\title{
Versatile interactions and bioinformatics analysis of noncoding RNAs
}

Q. Chen, X. Meng, Q. Liao, M. Chen*

Department of Bioinformatics, College of Life Sciences, Zhejiang University, Hangzhou, P. R. China

*e-mail:mchen@zju.edu.cn

Key words: noncoding RNAs, ncRNA transcription, ncRNA-RNA interaction, ncRNA-DNA interaction, ncRNA, protein interaction, bioinformatics resources

Advances in RNA sequencing technologies and computational methodologies have provided a huge impetus to noncoding RNA (ncRNA) study. Once regarded as inconsequential results of transcriptional promiscuity, ncRNAs were later found to exert great roles in various aspects of biological functions. They are emerging as key players in gene regulatory networks by interacting with other biomolecules (DNA, RNA or protein). Here, we provide an overview of ncRNA repertoire and highlight recent discoveries of their versatile interactions. To better investigate the ncRNA-mediated regulation, it is necessary to make full use of innovative sequencing techniques and computational tools. We further describe a comprehensive work- flow for in silico ncRNA analysis, providing up-to-date platforms, databases and tools dedicated to ncRNA identification and functional annotation. 